\title{
Analyzing and Geo-visualizing Individual Human Mobility Patterns Using Mobile Call Records
}

\author{
Chaogui Kang*, Song Gao*, Xing Lin*, Yu Xiao*, Yihong Yuan ${ }^{\dagger}$, Yu Liu*+ and Xiujun $\mathrm{Ma}^{\ddagger+}$ \\ *Institute of Remote Sensing and Geographical Information Systems, Peking University, \\ Beijing, China 100871 \\ ${ }^{\dagger}$ Department of Geography, University of California, \\ Santa Barbara, CA 93106, USA \\ ${ }^{\ddagger}$ Key Laboratory of Machine Perception (Minister of Education), Peking University, \\ Beijing, China 100871 \\ +Corresponding author:liuyu@urban.pku.edu.cn,maxj@cis.pku.edu.cn
}

\begin{abstract}
Understanding the dynamics of the individuals' daily mobility patterns is very important in a wide range of fields. However, lacking of tools to monitor the time-resolved location of individuals makes this research consume both tremendous time and money in the past. Nowadays, the rapidly developing ability to collect space-time activity (STA) data through new information technologies such as cellular phones, WiFi, and GPS methods is improving the quantity and quality of these data and reducing their cost. Also, "People-Based GIS" gives us a new perspective for analyzing and visualizing these data. In this paper, we introduce several preprocessing and spatiotemporal analysis methods for such new ICT data in individual human mobility patterns mining and urban analysis under the time geography framework. By using millions of raw mobile call records of a large city in China, we compute statistical characteristic of cell phone usage both at different time of a day and on different days of a week to derive aggregated mobility patterns for millions of mobile phone users and individual mobility patterns for different groups which are divided by some selected social factors, such as gender and age. The research methods and results also illustrate how to make 3D or 2D graphic representations of individual activities patterns and their evolution through space and time.

Keywords-individual mobility patterns; mobile call records; spatio-temporal data mining; geo-visualization; time geography
\end{abstract}

\section{INTRODUCTION}

Understanding the daily dynamics of individuals is very important in a wide range of fields, such as accessibility studies, location-based services, urban planning and crisis management [1]-[4]. Much research on the individual human mobility patterns is conducted using travel diary datasets that are collected by census and questionnaire ("time-space diary") [3], [4]. However, these approaches consume both tremendous time and money. More disappointedly, it is very difficult to gain enough amount of sample data and ensure their accuracy using these methods. Hence, more powerful data collecting techniques are needed in order to collect data with better accuracy and higher representativeness, so that we can gain more insights into people's mobility patterns. Fortunately, due to the remarkable improvement in Information Communication Technology (ICT), the technique for determining the geographic location of cell phones and other handheld devices is becoming increasingly available and providing a better choice or solution.
As a hot topic in the information communication industry in recent decades, the application of mobile phone location is opening the way to a wide range of applications, collectively referred to as location-based services (LBS). It is becoming a new and very promising data collection approach to analyze individual and urban mobility on a large-scale [5], and making studies that primarily aim at individual easy and prevalent [1]. Considering that the traditional static spatial frameworks based on fixed points (e.g., home or workplace) and distances among them are no longer adequate for understanding urban travel and individual mobility patterns, mobile call data can provide detailed records and offer novel avenues to map and explore individual human activity-travel and social communication networks [3], [4]. Hence, being aggregated and relatively accurate data in cities, the mobile call data can be used as a powerful tool for understanding human mobility and urban analysis.

In this paper we introduce several preprocessing and spatiotemporal analysis methods for such new ICT data in individual human mobility patterns mining and urban analysis. Our study is based on the geographical mapping and the statistical analysis of data derived from cell phone usage at different time of a day and over different days. We aim to examine the mobility patterns of millions of mobile phone users, particularly, the differences of the individual human mobility patterns of subgroups by certain social attributes/factors, allowing us to thoroughly study how these factors (such as gender and age) influence the spatial and temporal patterns of an individual human's travel activities, or to find out the relationships between them.

\section{RELEVANT RESEARCH ON GEO-VISUALIZING AND ANALYZING HUMAN ACTIVITY PATTERNS}

Due to the rapidly development in hardware and software for computer graphics and the increasing availability of georeferenced data, modern cartographic visualization is evolving from traditional map-making into a new paradigm of geovisualization [6]. By utilizing the power of human vision, many previous studies have demonstrated the effectiveness of geo-visualization in spatial data exploration, hypothesis 
generation, and knowledge construction as it can provides theories and techniques for a wide range of research and application fields [7]-[11]. Being more intuitively and expressive, the geo-visualization approach allows more realistic and detailed depictions of human activities in space-time. Hence, it has been widely applied to study diverse locationdependent phenomena and fruitful research results have been obtained [12], [13]. Within the current paradigm of geovisualization laid out by previous researchers [11], [12], this section reviews the major geo-visualization issues, solutions, and their evolution through time.

\section{A. Geo-visualizing Human Trajectories in Space-Time}

How to represent people's geographic locations and spatial movement throughout the day is one of the major tasks in geovisualizing an individual's daily activities. In the early studies, the animation approach and interactive methods (temporal brushing and temporal focusing) are often applied to reflect dynamic geographic phenomena on the temporal dimension by visualizing frames of animation sequentially [14]. This, however, is a drawback for studying human activity-travel behavior since an individual frame is only a snapshot in time and connections between activities and travel are not explicit when using these methods. Progressively, an approach using separate but linked views of the spatial and temporal dimensions (such as the space view and time view [13]) is introduced. This approach allows for an in-depth analysis of a particular dimension, but does not allow for the entire pattern to be visualized. Additionally, several other major approaches also have been proposed to present space and time in GIS, including object-oriented approach [15], [16], eventbased approach [17], and three-domain model [18]. Although these approaches demonstrate various ways to represent space and time in GIS, they are not good candidates to efficiently and effectively represent space-time constraints.

In this regard, the time-geography perspective provides an excellent framework for illustrating how a person navigates his/her environment in space-time and how his/her daily activities are often governed by three types of spacetime constraints [19]. Drawing upon this perspective, people's movement can be portrayed as space-time paths in a 3D "aquarium" in which the $\mathrm{X}$ and $\mathrm{Y}$ axes represent the geographic locations of activities and the $\mathrm{Z}$ axis represents the temporal dimension in the form of a 24-hour day [20]-[22]. The advantage of this approach is its ability to simultaneously represent the spatial and temporal characteristics of people's movement. However, it is difficult to show several important attributes of people's activity-travel behavior at the same time. To accommodate the multi-dimensional activity attributes in exploratory data analysis, a variety of bivariate and multivariate visualization techniques have been developed, including the scatter plot matrix [23], parallel coordinate plots [24], [25], multi-dimensional scaling [26], [27], grant tours [28], star glyphs [29], and Chernoff faces [30]. In addition, representing a large number of individual space-time paths may lead to an unclear view as the space-time paths may tangle together. Hence, deriving aggregate patterns of subgroups by certain nature-social factors illustrates a more clear view and meaningful interpretation on human mobility patterns.

\section{B. Analyzing Human Mobility in Space-Time}

Time geography [19] and space-time sequence analysis are two generally accepted but different methodologies/frameworks for understanding human activity participation under constraints in space and time. Time-geography framework has a well-defined structure. However, partially due to the limited computational power in the past, it was used primarily as a conceptual model in most studies with limited progress in implementation with computational models [20], [31]. In addition, rigorous, analytical definitions of basic time geography entities and relationships do not exist for long time [31]. This limits abilities to make statements about error and uncertainly in time geographic measurement and analysis. It also compromises comparison among different time geographic analysis and the development of standard time geographic computational tools. To overcome this problem, Miller [31] conceived the time geographic measurement theory, which consists of analytical formulations for basic time geography entities and relations, specifically, the spacetime path, prism, composite path-prisms, stations, bundling and intersections. Recently, several more attempts have been made to use GIS for measurements and analysis of individual mobility under special space-time constraints [32], [33].

Space-time sequence analysis accounts for the fact that data points (people's locations) taken over space and time may have an internal structure such as autocorrelation, trend or seasonal variation [34], [35]. Indeed, although we rarely perceive any of our actions to be random, from the perspective of an outside observer who is unaware of our motivations and schedule, our activity pattern can easily appear random and unpredictable. Hence, many current models of human activity are fundamentally stochastic from Erlang's formula used in telephony to Lévy-walk models describing human mobility. However, González et al. (2008) found out that, in contrast with the random trajectories predicted by the prevailing Lévywalk models, human trajectories show a high degree of temporal and spatial regularity, each individual being characterized by a time-independent characteristic travel distance and a signification probability to return to a few highly frequented locations [36]. Similarly, by measuring the entropy of each individual's trajectory, Song et al. (2010) demonstrated that a $93 \%$ potential predictability in user mobility across the whole user base [37]. These studies indicate: despite the diversity of people's travel history, humans follow simple reproducible patterns. This inherent similarity in travel patterns could impact all phenomena driven by human mobility, from epidemic prevention to emergency response, urban planning and agent-based modeling [36], [37].

\section{Study AREA AND DATA Collection}

The study area of this research is the urban area of a city in China. It has direct jurisdiction over 8 districts, 5 county-level 
cities and 7 counties, covering an area of $53,100 \mathrm{~km}^{2}$. The total population of the city is about 9 million, including roughly 5 million urban and 5 million rural, respectively accounting half of the population. For the purpose of experiment, we randomly selected a set of 869,992 anonymously users from those making or receiving at least one phone call in the study area throughout 9 consecutive days, from Sunday to Monday of next week. The dataset was originally collected by a Chinese mobile phone carrier for billing and operational purposes, and was finally translated to 617 recorded positions by geocoding. It contains the call type (server / opposite), date, time, duration and coordinates (latitude / longitude) of the phone tower routing the communication for each phone call dialed or received by users and their basic information, such as gender and age. Furthermore, we only know the coordinates of the tower routing the communication, hence exact location of users are not known within a tower's service area. In our dataset, each tower serves an area of approximately $1 \mathrm{~km}^{2}$. In addition, we removed all jumps that took users outside the continental territory, and did not impose any additional criterion regarding the calling activity to avoid possible selection biases in the mobility pattern.

\section{GEO-VISUALIZING AND ANALYZING INDIVIDUAL MOBILITY PATTERNS}

\section{A. 3-D and 2-D Space-Time Paths Modeling}

Representation plays an important role in problem solving using a computer system. To efficiently and explicitly represent individual's activity-travel under space-time constraints, we represent and organize the call data both in 2D and 3D. In our research, the geo-visualization of 3D and 2D space-time paths were developed using Python integrated ArcScene and ArcMap.

To simulate the individual's movements in space-time, we adopt an orthogonal 3D coordinate system, which 2D for space and $1 \mathrm{D}$ for time. As defined in the space-time framework, a spatiotemporal point feature is represented with a triplet of $\langle x, y, z\rangle$, where $x$ and $y$ specify a location on a $2 \mathrm{D}$ space and $t$ indicates a specific time. A spatiotemporal line feature is represented as a sequence point features along the time dimension [20]. If an individual does move in space over a period of time, his/her path will appear as a vertical line between the starting and ending times. While, if an individual moves between two locations, it will represent a sloped line in the three-dimensional space-time between the two locations [19]. Under this 3D framework, we can qualitatively find out the differences between individual mobility patterns, which depict different space locations and time rhythm.

Figure 1 illustrates the visualization of individual human trajectories in space and time. Each color represents a single person. The time-budget constraint that work hours or job imposes on the activities of individuals is evident. The long work hours are revealed by the long activity duration at fixed location, as indicated by the length of the vertical segments of the space-time paths during the day. On the contrary, people with shorter work hours are indicated by the short vertical daytime segments of their space-time paths shown in Figure 1. This, in some extent, reflects the differences in people's lifestyles and professions.

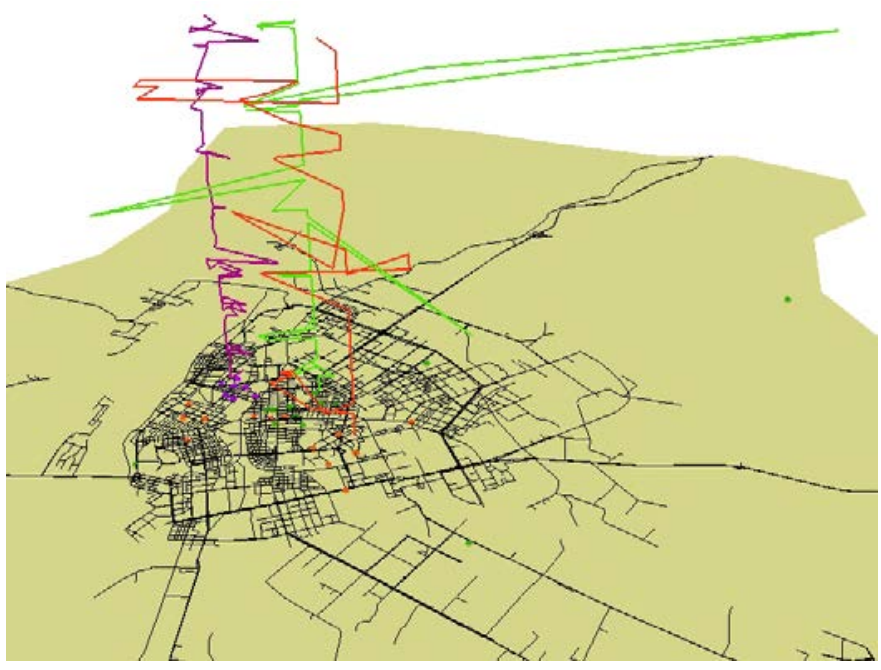

Figure 1. Visualization of individuals' trajectories.

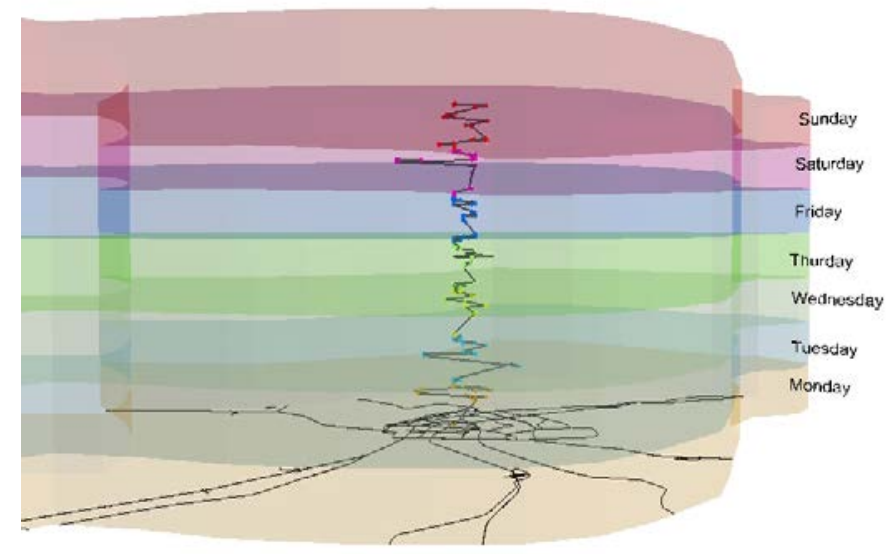

Figure 2. A week-long individual travel-activity path.

Figure 2 illustrates individual's movement through one week. Every individual has a daily activity program consisting of a number of out-of-home activities, including activities that are spatially and/or temporally fixed for certain individuals (e.g., workplace) and others that can be undertaken at various locations or times of the day (e.g., grocery stores). The former type of activities usually serves as "pegs" in the daily spacetime trajectory of the individual, and is often referred to as "fixed activities" [38]. Hence, for working people, their activities show a high degree of regularity in space and time on workdays, but distinct change on weekends, which can be explained by the fact that the working people prefer to go to a new place for recreation or go on tourism. In other words, their space and time constraint allows for only a few 


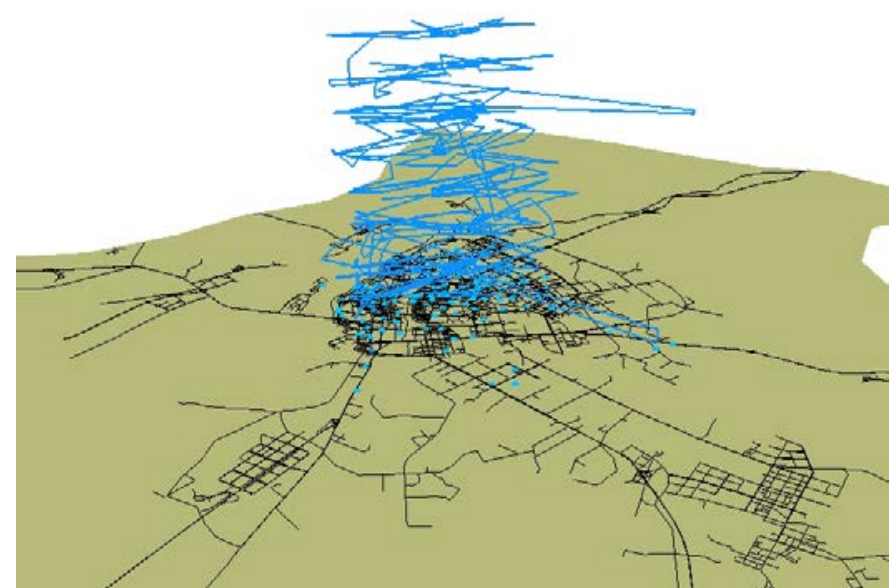

Figure 3. Irregular individual trajectory in space.

nonemployment activities to be undertaken, besides a meal or some short personal business stops near the workplace at lunch time. Figure 3 reveals a totally different activity patterns of individual human. As they tend to perform more nonemployment activities during the day, less restrictive timebudget constraint is given and their space-time paths appear to be more fragmented during the weekdays than those of the people that are full-timely employed.

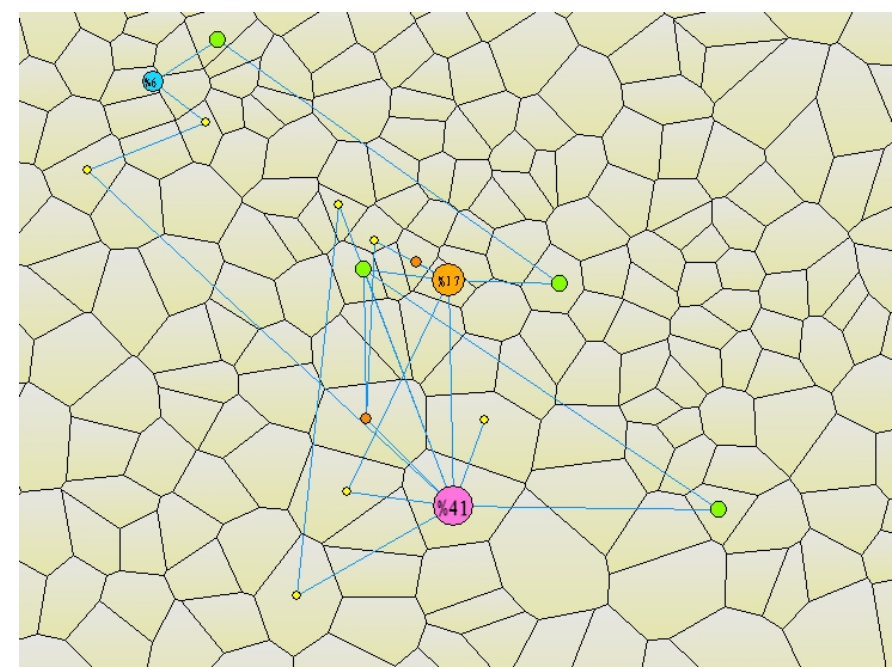

Figure 4. Mobility network associated with a mobile phone user who usually appears at fixed locations.

In order to represent the mobility patterns in a more quantitative and meaningful manner, a 2D trajectory modeling approach is introduced in this study. In this method, each dot corresponds to a mobile phone tower, and each time a user makes a call, the closest tower that routes the call is recorded, pinpointing the user's approximate location. The gray colored lines represent the recorded movement of the user between the towers. The size of the dots corresponds to the frequency of calls the user made in the vicinity of the respective tower, and the widths of line edges are proportional

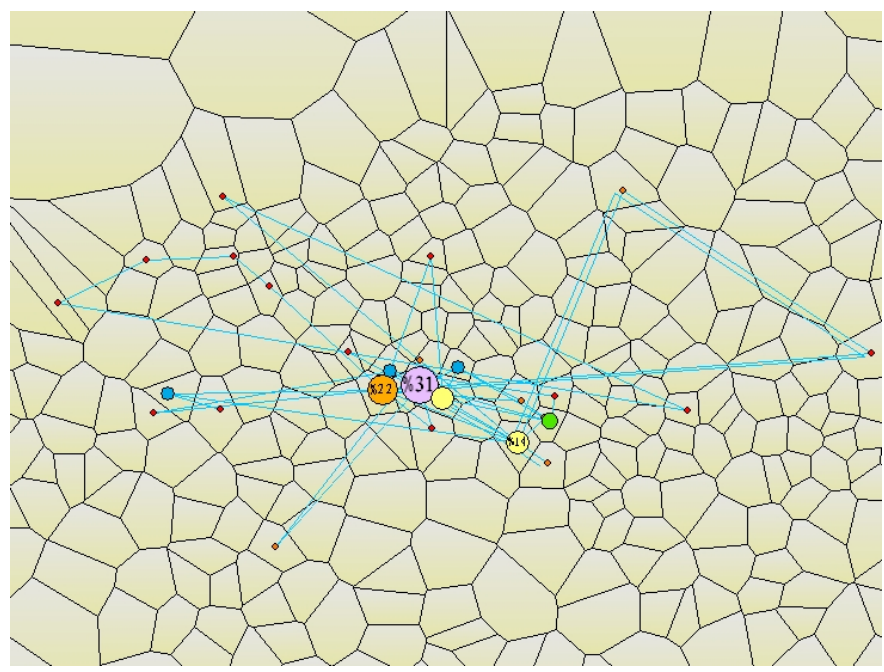

Figure 5. Mobility network associated with a mobile phone user who usually visits few fixed places on fixed time repeatedly and appears at a lot of locations occasionally.

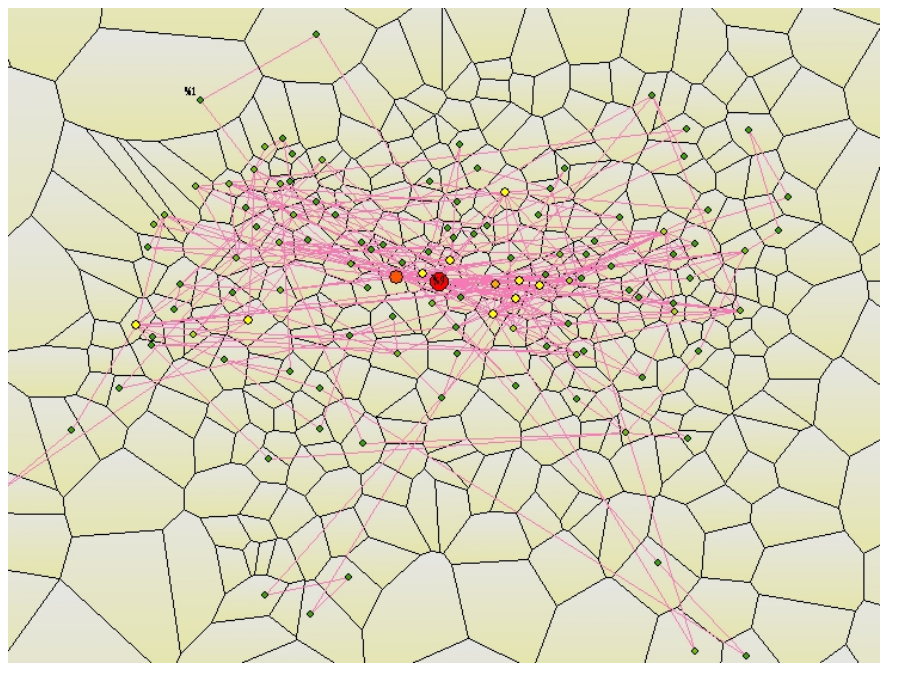

Figure 6. Mobility network associated with a mobile phone user who usually moves across a large set of locations and seldom visits the same place repeatedly.

to the frequency of the observed direct movement between two towers [36]. Figure 4 represents a week long activity-travel pattern of some person who usually moves to or appears at some fixed locations. It tells that some people's trajectories show a high degree of temporal and spatial regularity, each individual being characterized by a time-independent characteristic travel distance and a high probability to return to a few highly frequented locations, which are usually home and work places. Figure 5 represents a similar activity-travel pattern. But these people appear to be more floating. They visit few fixed places at fixed time repeatedly, and appear at a lot of locations occasionally. In this regard, their behaviors are less predictable. Figure 6 represents a different type of individual activity-travel pattern. These people's activities are fundamentally stochastic and unpredictable. They move across a large set of locations and seldom visit the same place repeatedly. In our research, these people are supposed to be 
outdoor workers and HOBOS (people who don't have a regular job).

\section{B. Distribution of Individual's Travel-distance}

Space and time are two essential factors that serve as constrains on planning and carrying out human activities. Every activity has its specific characteristics in space and time. The differences in people's daily movements can be better explained through corresponding geographical distribution. In our research, we implement the radius of gyration to describe the typical range of a user's trajectory. Figure 7 presents the distribution of the typical distance covered by each of the 869,992 users. It demonstrates that as the travel distance increases, the population increases correspondingly first. But when the travel distance comes to a particular value (it is 2 $\mathrm{km}$ in our research), the population decreases as it increases. Finally, the population keeps steady at a low level. Figure 8 depicts that more than $95 \%$ of the population's travel distance are within $10 \mathrm{~km}$. Obviously, the distribution of individual's travel distance indicates: since a long travel usually costs a lot of time and money, the majority of mobile population travels a modest distance, neither too near nor too far. To some extent, the distribution of individual's travel distance also reflects the city size and variations in residents' living standards and their transportation.

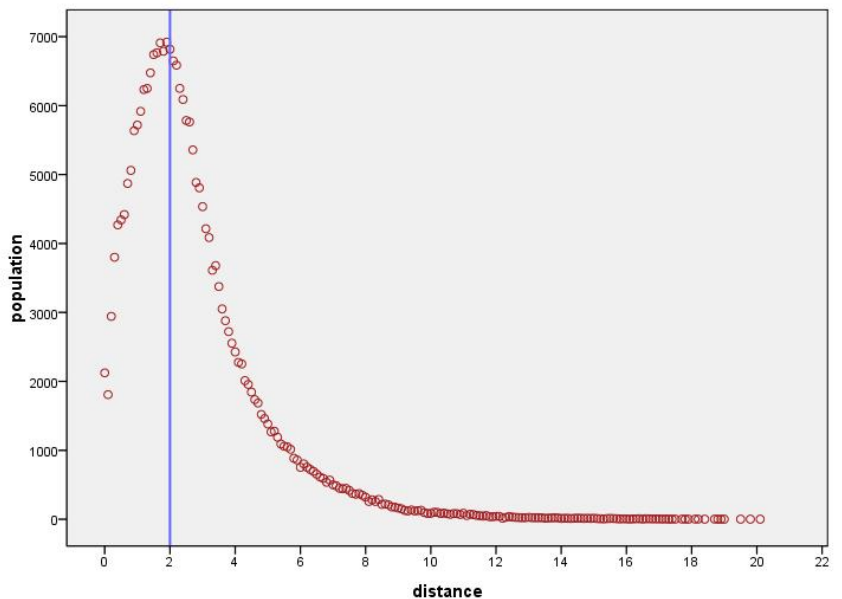

Figure 7. The distribution of the typical distance covered by each of the 869,992 users.

\section{Characterizing Subgroups by Age, Gender and Call-times}

Recent debate on gender/age differences in access to employment opportunities raises concerns about the difficulties in using the length of the commute trip as a measure of individual human mobility. As either long or short work trips can be taken as indicators of spatial inequality in the access to employment, it is recognized that "correct" interpretations of results depend on a contextualized understanding of the life situations of the individuals being studied [3]. Based on the actual condition of the study area and the data constraints, the samples are classified by their gender and age.

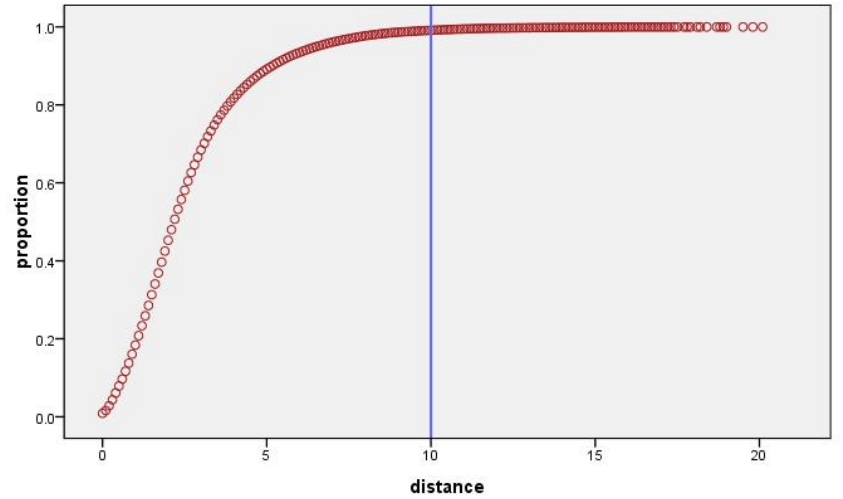

Figure 8. The proportion of mobile phone users covering different distance.

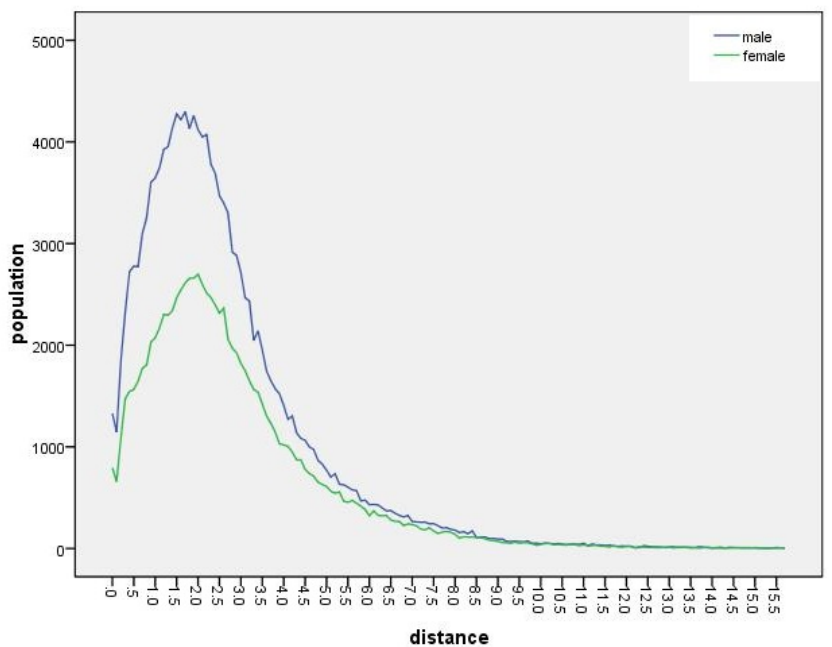

Figure 9. The distribution of the travel distance covered by man and women

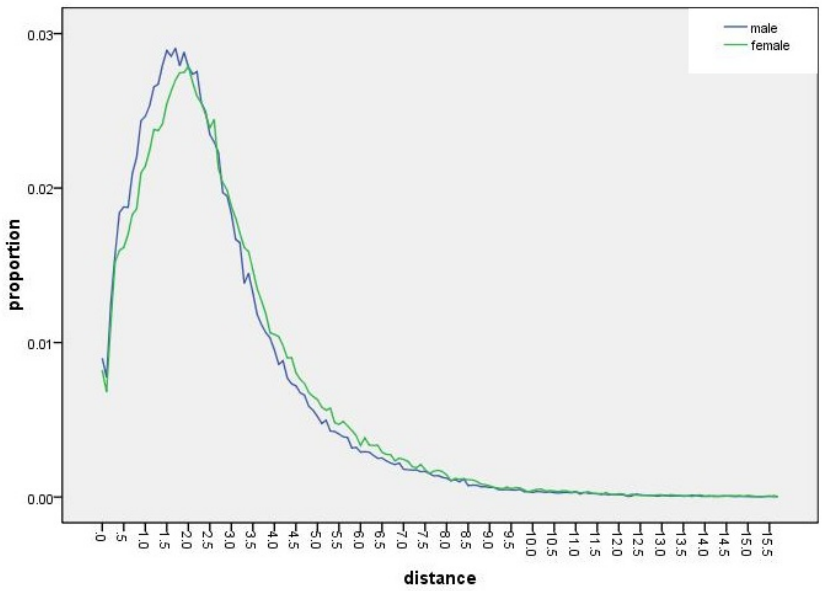

Figure 10. The proportion of mobile phone users covering different distance divided by gender.

The samples are divided into two groups, male and female, to examine gender differences in mobility patterns at first. Then, the travel distance were computed for each individual in the two subsamples. Figure 9, 10 indicate that men's and women's travel distances both depict a statistical regularity as shown in Figure 7. This also suggests that the length of the 
commute trip may not be an appropriate indicator of how much constraint an individual faces. Hence, much future research is needed to decipher the complex relationship between access to urban opportunities (including jobs) and the length of the commute trip.

Concerning the factor of age, the samples are divided into four groups: adolescent (age $<20$ ); youth (age $20 \sim 39$ ); middle-aged (age $40 \sim 59$ ); old (age $>60$ ). Figure 11, 12 illustrate that a larger proportion of the adolescent and the elderly prefer to stay at one place or just go out for a walk in the surrounding area, which are indicated by the large proportion of the population traveling short distance. It also suggest that the youth and the middle-aged usual travel farther than them, and their activity are highly heterogeneous, which is indicated by the distribution of these two subgroup's travel distances in Figure 11, and can be explained by the fact that: adolescent and old people perform more non-employment activities and make less out-of-home trips (e.g., working, serve passengers, shopping) than that the youth and the middle-aged do. Such activities constitute an important dimension of age differences in everyday life.

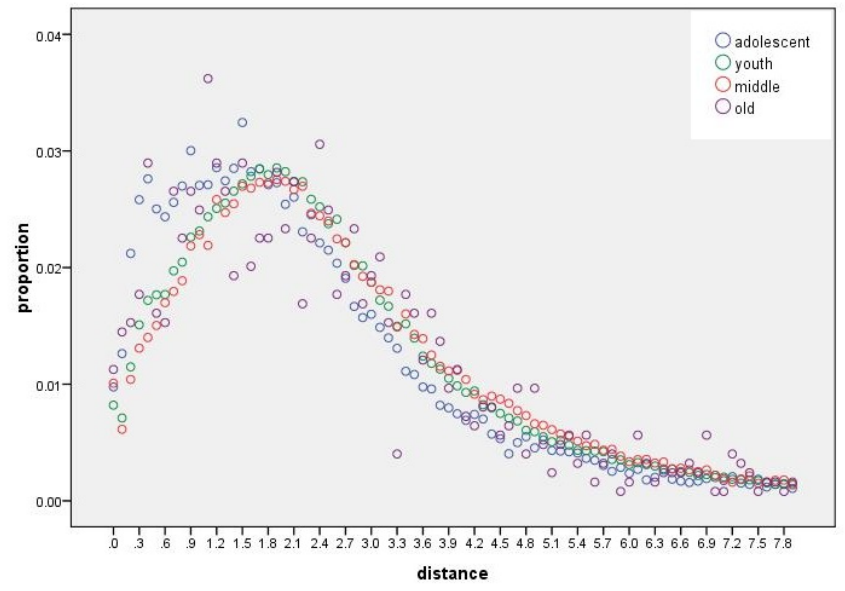

Figure 11. The distribution of the travel distance divided by age.

Many previous studies have shown the particularly strong correlation between mobile phone use and activity-travel behavior [39], [40]. Communication patterns are known to be highly heterogeneous: some users rarely use the mobile phone while others make hundreds or even thousands of calls each month. Hence, to characterize the dynamics of individual communication activity, we grouped users based on their total number of calls. For each user we measured the variation of his/her number of calls between the 9 sampled consecutive days. To examine the assumption about the relationship between telecommunications and transport on the level of individual persons, we calculate the average travel distance of each group. The result (see Figure 13) demonstrates that the larger the number of calls is, the longer the average distance of the group becomes.

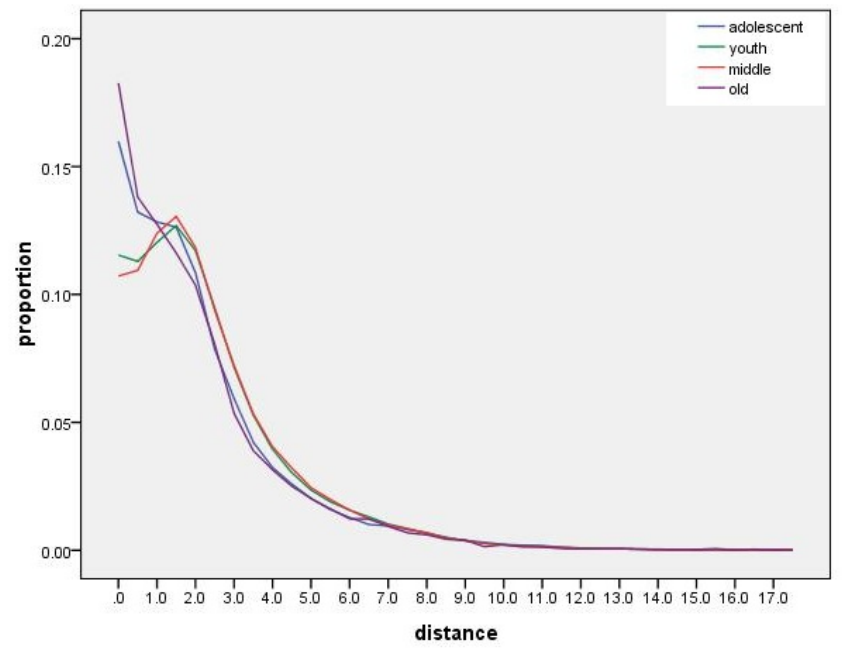

Figure 12. The proportion of mobile phone users covering different distance divided by age.

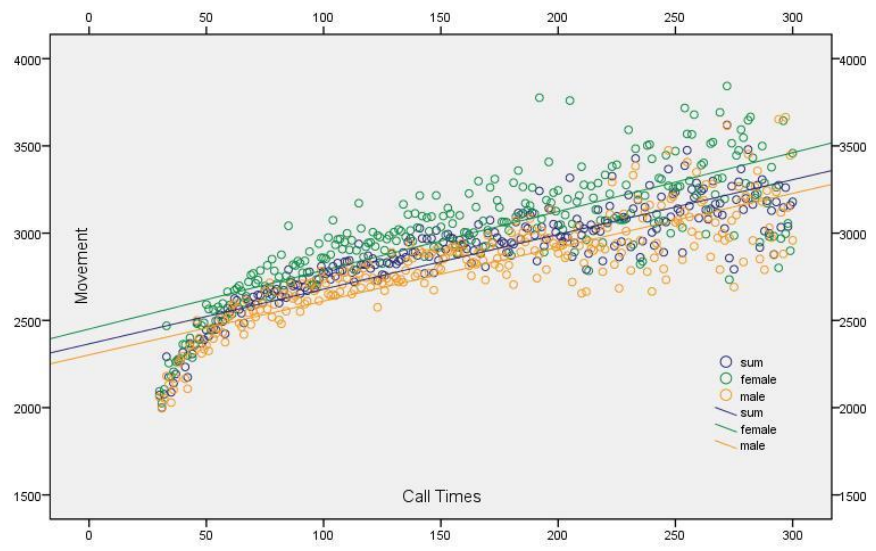

Figure 13. The correlation between mobile phone usage and travel behavior.

\section{CONCLUSion}

The article presented a study about the preprocessing and spatiotemporal analysis methods for the new ICT (Information Communications Technology) data in individual human mobility patterns mining and urban analysis. As to telecommunications use, a focus was laid on the use of the mobile phone in this paper. By using millions of raw mobile call records of a large city in China, we computed statistical characteristic of cell phone usage both at different time of a day and on different days of a week to derive aggregated mobility patterns for millions of mobile phone users in the study area and individual mobility patterns for different groups which are divided by some selected social factors, such as gender and age. In our research, we also implemented an effective measurement "the radius of gyration", which is an indicator of individual's mobility, to portray human activities in physical space and characterize the individual mobility pattern. The research results showed that: within the collected samples, the individual's activity distance usually varies within a reasonable range; there are no significant gender differences in travel 
distance for women and men; the youth's and the middleaged's travel distance and activeness are generally higher than those of the adolescents and the elderly. Considering the fact that the chosen city of case study is a typical one in China and the high prevalence rate (about 50\%) of mobile phone usage within Chinese people, the above results have potential value for a more general case beyond the collected samples. The research methods and results shown in this paper also depict a way to make 3D or 2D graphic representations of individual activities patterns and their evolution through space and time.

\section{REFERENCES}

[1] C. Ratti, D. Frenchman, R. M. Pulselli and S. Williams. "Mobile Landscapes: Using Location Data from Cell Phones for Urban Analysis". Environment and Planning B: Planning and Design. 2006, vol.33, pp.727748.

[2] R. Ahas and U. Mark. "Location Based Services: New Challenges for Planning and Public Administration?". Futures. 2005, vol.37, pp.547-561.

[3] M. P. Kwan. "Gender, the home-work link, and space-time patterns of nonemployment activities". Economic Geography. 1999,vol.75, no,4, pp.370-394.

[4] M. P. Kwan. "Gender and Individual Access to Urban Opportunities: A Study Using Space-Time Measures". Professional Geographer. 1999, vol.51, no.2, pp.210-227.

[5] P. Mateos and P. F. Fisher. "Spatiotemporal accuracy in mobile phone location: assessing the new cellular geography". In J. Drummond, R. Billen, D. Forrest and E. João (eds.), Dynamic \& Mobile GIS: Investigating Change in Space and Time. Taylor \& Francis, London, pp.189-212. ISBN: 0-8493-9092-3.

[6] F. Ren and M. P. Kwan. "Geovisualization of Human Hybrid ActivityTravel Patterns". Tansactions in GIS. 2007, vol.11, no.5, pp.721-744.

[7] M. Gahegan and B. Brodaric. "Computational and visual support for geographic knowledge construction: Filling in the gaps between exploration and explanation". In Proceedings of the Tenth International Symposium on Spatial Data Handling, Ottawa, Canada. 2002, pp.11-14.

[8] M. J. Kraak. "Geovisualization illustrated". ISPRS Journal of Photogrammetry and Remote Sensing. 2003, vol.57, pp.390-399.

[9] E. L. Koua and M. J. Kraak. "Integrating computational and visual analysis for the exploration of health statistics". In P. F. Fisher (eds.) Advances in Spatial Data Handling II: Proceedings of the Eleventh International Symposium on Spatial Data Handling. New York, SpringerVerlag. 2004, pp.653-664.

[10] E. L. Koua and M. J. Kraak. "Geovisualization to support the exploration of large health and demographic survey data". International Journal of Health Geographics. 2004, vol.3, pp.12.

[11] A. M. MacEachren, M. Gahegan, W. Pike, I. Brewer, G. Cai, E. Lengerich and F. Hardisty. "Geovisualization for knowledge construction and decision-support". Computer Graphics and Applications. 2004, vol.24, pp.13-17.

[12] A. M. MacEachren and M. J. Kraak. "Exploratory cartographic visualization: Advancing the agenda". Computers and Geosciences. 1997, vol.23, pp.335-343.

[13] D. Mountain. "Visualizing, querying and summarizing individual spatiotemporal behaviour". In J. A. Dykes, A. M. MacEachren, and M. J. Kraak (eds.) Exploring Geovisualization. Amsterdam, Elsevier. 2005, pp.181200

[14] P. Laube, T. Dennis, P. Forer and M. Walker. "Movement beyond the snapshot-Dynamic analysis of geospatial lifelines". Computers, Environment and Urban Systems. 2007, vol.31, pp.481-501.

[15] M. F. Worboys. "A Model for Spatio-temporal Information". Proceedings: the 5th International Symposium on Spatial Data Handling. 1992, vol.2, pp.602-611.

[16] M. F. Worboys. "Object-oriented Approaches to Geo-referenced Information". International Journal of Geographical Information Systems. 1994, vol.8, pp.385-399.

[17] D. J. Peuquet and N. Duan. "An Event-based Spatiotemporal Data Model (ESTDM) for Temporal Analysis of Geographical Data". International Journal of Geographical Information Systems. 1995, vol.9, pp.7-24.
[18] M. Yuan. "Modeling Semantics, Temporal, and Spatial Information in Geographic Information Systems". In M. Craglia and H. Couclelis (eds.) Geographic Information Research: Bridging the Atlantic. London: Taylor and Francis. 1996, pp.334-347.

[19] T. Hägerstrand. "What about People in Regional Science?". Papers of the Regional Science Association. 1970, vol.24, pp.1-12.

[20] H. Yu and S. L. Shaw. "Exploring Potential Human Activities in Physical and Virtual Spaces: A Spatio-temporal GIS Approach". International Journal of Geographical Information. 2008, vol.22, no.4, pp.409-430.

[21] M. P. Kwan and J. Lee. "Geovisualization of human activity patterns using 3D GIS: a time-geographic approach". In M. Goodchild, D. Janelle (eds) Spatially Integrated Social Science: Examples in Best Practice. New York: Oxford University Press, Oxford. 2004, pp.48-66.

[22] B. Lenntorp. "Paths in space-time environments: A time geographic study of movement possibilities of individuals". Lund Studies in Geography Number 44, Royal University of Lund, Sweden, 1976.

[23] D. F. Andrews. "Plots of high-dimensional data". Biometrics. 1972, vol.29, pp.125-136.

[24] A . Inselberg. "The plane with parallel coordinates". The Visual Computer. 1985, vol.1, pp.69-91.

[25] A. Inselberg. "Discovering multi-dimensional structure using parallel coordinates". In Proceedings of the American Statistical Association, Section on Statistical Graphics, Washington, D.C.. 1989, pp.1-16.

[26] J. B. Kruskal and M. Wish. "Multidimensional Scaling". Beverly Hills, CA, Sage Publications. 1977.

[27] F. W. Young and R. M. Hamer. "Multidimensional Scaling: History, Theory and Applications". New York, Erlbaum. 1987.

[28] D. Asimov. "Grand tour". SIAM Journal of Scientific and Statistical Computing. 1985, vol.6, pp.128-143.

[29] S. Fienberg. "Graphical methods in statistics". The American Statistician. 1979, vol.33, pp.165-178.

[30] H. Chernoff. "The use of faces to represent points in k-dimensional space graphically". Journal of the American Statistical Association. 1973, vol.68, pp.361-368

[31] H. J. Miller. "A measurement theory of time geography". Geographical Analysis. 2005, vol.37, pp.17-45.

[32] S. L. Shaw and H. Yu. "A GIS-based Time-geographic Approach of Studying Individual Activities and Interactions in A Hybrid Physicalvirtual Space”. Journal of Transport Geography. 2009, vol.17, pp.141149.

[33] Z. Shen and K. L. Ma. "MobiVis: A Visualization System for Exploring Mobile Data". IEEE Pacific Visualisation Symposium. 2008, pp.175-182.

[34] N. Shoval and M. Isaacson. "Sequence Alignment as a Method for Human Activity Analysis in Space and Time". Annals of the Association of American Geographers. 2007, vol.97, no.2, pp.282-297.

[35] N. Shoval. "Tracking technologies and urban analysis". Cities. 2008, vol. 25 , pp.21-28.

[36] M. C. González, C. A. Hidalgo and A. L. Barabási. "Understanding individual human mobility patterns". NATURE. 2008, vol.453, pp.779782 .

[37] C. Song, Z. Qu, N. Blumm and A. L Barabási. "Limits of Predictability in Human Mobility". Science. 2010, vol.327, no.1018, pp.1018-1021.

[38] I. Cullen, V. Godson and S. Major. "The structure of activity patterns". In A. G. Wilson (eds.) Patterns and Processes in Urban and Regional Systems, pp.281-296. London:Pion. 1972.

[39] T. Schwanen and M. P. Kwan. "The Internet, mobile phone and spacetime constraints". Geoforum. 2008, vol.39, pp.1362-1377.

[40] C. Nobis and B. Lenz. "Communication and mobility behavior-a trend and panel analysis of the correlation between mobile phone use and mobility". Journal of Transport Geography. 2009, vol.17, pp.93-103. 